\title{
Warum wir Leibniz noch brauchen oder Der begehbare Kopf
}

\author{
Eike Christian Hirsch
}

\section{Es geht mir nur um eine strategische Überlegung}

Warum brauchen wir Leibniz noch? Mir scheint, wir stehen heute in einem Konflikt der Weltanschauungen, der stark an das erinnert, was Leibniz erlebt hat. Es geht und ging um die Frage, ob alles, was es gibt, Materie ist, oder ob der Geist ihr eigenständiges Gegenüber ist. Darum geht es auch heute. Der Physiologe Wolf Singer etwa (vom Max-Planck-Institut für Hirnforschung in Frankfurt am Main, seit langem medienwirksam präsent) bestreitet einen „Geist“. Er sagte etwa im Interview: „Alles, was wir in dualistischen Leib-Seele-Modellen gern dem Geistigen zuschreiben, ist rein biologisch bedingt“. Für Singer ist also alles „rein biologisch“, daher gibt es auch keine Willensfreiheit.

Leibniz hat zu seiner Zeit die Gegenposition auf eine Weise vertreten, die man als ziemlich robust bezeichnen kann. Eine Position, mit der er den Geist verteidigt hat gegen die Annahme, es gebe nur die Materie.

Doch ich will nicht sagen, dass seine Argumente alle richtig waren; auch seine Lösung ist nicht unbedingt richtig oder heute noch gültig. Behaupten will ich nur, dass er damals eine Verteidigungslinie aufgebaut hat, die ziemlich hart war, härter als die Position, die heute wohl ein Freund von Geist und Seele einnehmen würde. Und insofern ist er, was die Verteidigungsstrategie angeht, noch heute eine feste Burg.

\section{Die Frontlinie damals und heute}

Zunächst versuche ich zu skizzieren, in welcher Auseinandersetzung Leibniz stand. $\mathrm{Zu}$ seiner Zeit waren fast alle Forscher und Denker Anhänger von René Descartes, der fünfzig Jahre vor Leibniz geboren wurde. Er hatte die Welt bekanntlich geteilt in Materie und Geist, die er res extensa und res cogitans nannte. Er hielt die Materie und die ganze Natur für eine Mechanik, alles verhielt sich wie eine Maschine. Doch Descartes hatte noch am Geist festgehalten, der res cogitans. 
Zu Zeiten von Leibniz war ein Teil der Cartesianer, wie man seine Schüler nannte, der Meinung, alles sei Mechanik und Materie, man könne auf die Annahme eines Geistes oder einer Seele verzichten. Ja, man müsse auf den Geist verzichten, denn alles laufe in Wirklichkeit mechanisch und determiniert ab, eben wie in einer Maschine, determiniert sei damit auch alles, was in unserem Kopf vorgeht.

Leibniz hat schon in jungen Jahren erkannt, dass der Geist schwer gefährdet war. Doch warum lag ihm so viel an der Annahme, es gebe einen Geist? Was trieb Leibniz an? Er sah, dass letztlich der freie Wille nicht zu halten sein würde, wenn alles natürlich-mechanisch abläuft. Der freie Wille, das ist die Würde des Menschen. Ohne Geist aber kein freier Wille, ohne freien Willen keine Würde. So wird das auch heute noch gesehen: Wenn unsere Gedanken und Empfindungen reine Naturprodukte sind, gibt es keinen freien Willen.

Mein Zeuge ist wieder der bekannte WolfSinger, von dem wir schon die Ansicht gehört haben, alles, was im Kopf entsteht, sei reine Natur. Seine Konsequenz ist: Es gibt keinen freien Willen. Für diese These ist Singer besonders bekannt geworden. Willensfreiheit, sagt er, sei „inkompatibel mit dem, was wir über die Funktion unserer Gehirne gelernt haben“". Oder er sagt dasselbe mit anderen Worten: „Keiner kann anders, als er ist. Verschaltungen legen uns fest. Wir sollten aufhören, von Freiheit zu reden“".

Wolf Singer sieht es selbst am deutlichsten, dass gerade diese Erkenntnis die Würde des Menschen in Frage stellt. Keine Willensfreiheit! Singer sagt dazu: „Diese Verletzung unseres überlieferten Selbstverständnisses tut weh auch dem, der die Erkenntnisse zu Tage fördert". Also auch und zuerst dem Forscher Wolf Singer selbst.

\section{Der Gegenentwurf}

Leibniz hielt zu seiner Zeit dagegen, als durch Denker wie Hobbes oder Spinoza die Willensfreiheit bestritten worden war. Er kämpfte für den Geist und er brauchte ihn, wenn ich so sagen darf, um die Willensfreiheit zu retten.

Nun möchte ich Ihnen gleich das berühmte Gedankenexperiment vom begehbaren Kopf vortragen. Es wirkt erstaunlich modern und radikal. Aber zuvor muss ich noch auf den entscheidenden Schritt aufmerksam machen, den Leibniz tut, um den Geist so zu etablieren, dass er nicht wegdiskutiert werden kann.

Es gibt den Begriff Geist nämlich doppelt. Leibniz hat den umfassenden Begriff davon gewählt. Wir hingegen denken doch bei „Geist“ gewöhnlich 
an den menschlichen Geist, etwa wenn wir sagen: „All das hat der menschliche Geist hervorgebracht". Für Leibniz ist aber der Geist primär nicht das, was in unserem Kopf entsteht. Geist ist für ihn das, woraus der ganze Kosmos besteht, er ist also viel umfassender, fundamentaler gedacht. Der Kosmos ist Geist.

Was in unserem Kopf entsteht, ist nur eine Ausprägung dieses Geistes, ist vielleicht einfach nur die Art, wie der fundamentale Geist sich selbst erkennt. Unser Fühlen und Denken stammt aus diesem umfassenden, ursprünglichen Geist und ist von allem Materiellen zunächst einmal geschieden.

Das ist eine ziemlich radikale Ansicht, denn gewöhnlich meinen wir ja, unsere Gedanken und Empfindungen entstünden - irgendwie - im Gehirn aus biochemischen Vorgängen.

\section{Der begehbare Kopf}

Nun komme ich zum angekündigten Gedankenexperiment. Es findet sich in Leibniz' bekanntester Darstellung seiner Philosophie, in seinem Alterswerk, der so genannten Monadologie (Abschnitt 17). Leibniz denkt sich da einen begehbaren Kopf. Und ich muss wohl nur kurz darauf hinweisen, dass heute das Gehirn ja tatsächlich gleichsam betreten werden kann, weil die Wissenschaft mit Hilfe der Kernspintomografie dem Gehirn bei seinen Gedanken und Entschlüssen längst zusieht.

In etwas freier Übersetzung aus dem (französisch verfassten) Original lautet das Bild so: „Man stelle sich vor, dass es eine Maschine gäbe, die aufgrund ihrer Bauart denken, fühlen und wahrnehmen könnte. Diese Maschine kann man sich dann vergrößert so vorstellen, dass man in sie wie in eine Mühle eintreten könnte. Dann würde man in ihr nur Teile finden, die einander stoßen, und nichts, was die Leistung einer, Wahrnehmung ' zu erklären vermöchte“.

Dieses Gedankenexperiment ist erstaunlich modern, schon weil Leibniz die heutigen Untersuchungsmethoden gleichsam vorwegnimmt.

\section{Die Deutung dieses Bildes}

Noch erstaunlicher aber ist, dass er zu dem gleichen Schluss zu kommen scheint, den heute auch Gehirnforscher gern ziehen. Sie sagen dann, wie scheinbar auch Leibniz: „Wir sehen nur die Synapsen feuern, also die Bewegung von Molekülen, aber von einem Geist sehen wir nichts“. So ähnlich 
sagt es auch Leibniz: „Dann würde man in ihr nur Teile finden, die einander stoßen, und nichts, was die Leistung einer, Wahrnehmung' zu erklären vermöchte“.

Leibniz hat also schon gewusst: Wer das Gehirn betritt, der wird den Geist nicht finden. Und wir können nun zu den Gehirnforschern sagen: „Warum wundert ihr euch? Ihr werdet nur Teile finden, die einander stoßen. Das wusste schon Leibniz. Das ist kein Argument gegen den Geist".

Die Übereinstimmung zwischen Leibniz und manchen Naturwissenschaftlern besteht natürlich nur auf den ersten Blick. Beide sehen den Geist nicht - aus ganz gegensätzlichen Gründen.

Wir haben Aussagen von Singer gehört. Auch andere Forscher sind so weit gegangen zu sagen: „Was ,Ich“ oder ,Bewußtsein` genannt wird, gar "Geist', ist eine allzu subjektive Deutung rein biochemischer Abläufe“.

Ein wenig erinnert diese Position an den naturwissenschaftlichen Materialismus aus dem 19. Jahrhundert. Einer ihrer Vertreter, der niederländische Physiologe Jakob Moleschott (1822-1893), bestand grundsätzlich darauf, die geistig-seelischen Vorgänge seien körperlicher Art. Ihm wird der Satz nachgesagt, das Gehirn produziere seine Gedanken wie die Niere den Urin. Ebenso gern wird der geistreiche Kommentar eines Kollegen von Moleschott zitiert: „Wenn man den Kollegen Moleschott so reden hört, könnte man fast meinen, er hätte recht“.

\section{Wie Leibniz sein Gedankenexperiment verstanden wissen wollte}

Doch zurück zu Leibniz. Er entscheidet sich dafür, anzunehmen, dass wir, könnten wir das Gehirn betreten, darin nichts Geistiges erkennen könnten. Er sagt das nicht, um den menschlichen Geist zu leugnen, sondern im Gegenteil, um ihn zu stärken.

Unsere Gedanken und Empfindungen sind für Leibniz nämlich gar nicht das Produkt der physiologischen Vorgänge im Gehirn, sie sind vielmehr unabhängig von körperlichen Vorgängen. Unser Geist lebt - ganz im Gegenteil - aus dem anderen, dem großen Geist, aus dem das Universum besteht.

Wenn Sie mich richtig verstanden haben, so werden Sie verwundert sein. Und doch ist es so: Leibniz leitet unseren Geist nicht aus den körperlichen Vorgängen im Gehirn ab. Für ihn sind unsere geistig-seelischen Regungen unmittelbar Ausfluss des Geistes, aus dem die Schöpfung besteht. Das lehrt seine berühmte und doch so schwer verständliche Annahme einer „prästabilierten Harmonie" zwischen Körper und Geist. 
Leibniz' Auffassung ist, zugegeben, etwas radikal: Unsere Gedanken und Empfindungen entstehen gar nicht aus gehirnphysiologischen Prozessen.

\section{Die üblichen Konzessionen der Freunde des Geistes}

An dieser Stelle möchte ich kurz die Art betrachten, wie heutzutage gelegentlich die Annahme, es gebe einen Geist, begründet wird.

Ja, was tun eigentlich wir, die wir vielleicht gewöhnliche Freunde des Geistes sind und etwas von der Selbständigkeit des Geistes retten wollen, angesichts der überwältigend erfolgreichen Naturwissenschaft? Einer Wissenschaft also, die alles aus der Biochemie ableitet und uns leichthin erklärt, den Begriff Geist nicht zu brauchen? Nun, wir kleinlauten Freunde des Geistes versuchen es mit einem Kompromiss. (Und Sie ahnen es schon, ich will Ihnen zeigen, dass solch ein Kompromiss auf einer schiefen Ebene errichtet wird und nicht lange zu halten ist.)

Unsere gewöhnliche, durchaus vermittelnde Haltung beginnt mit der Konzession, dass wir sagen: „Ja, die Synapsen feuern, und aus ihrer Aktivität entstehen unsere Gedanken, unsere Empfindungen und Entscheidungen“. Das räumen wir ein. Aber wir fügen hinzu, um den Geist noch irgendwie zu retten: „Diese biochemischen Prozesse sind derart komplex und vielfältig, dass sie etwas hervorbringen, was von neuer Qualität ist. Aus der unglaublichen Quantität der vielen parallelen Prozesse entsteht eine neue Qualität, die wir den ,Geist" nennen“. Oder wir verwenden ein anderes Bild, um den Unterschied von organischen Vorgängen und geistigem Produkt anschaulich zu machen: „Es vollzieht sich hier eben die Sublimierung des Materiellen, mit der Folge, dass sich etwas ganz Neues zeigt, und das ist der Geist. Geist, das ist gleichsam sublimierte Materie“.

Diese Einstellung ist ehrenvoll, aber ich finde, dass sie sich kaum halten lässt. Zu nahe liegt es, zu entgegnen, dass ein Produkt aus materiellen Vorgängen ein materielles Produkt bleibt. Und dass der Wunsch, nun von Geist (oder gar Seele) zu sprechen, eher unser romantisches Bedürfnis befriedigt, als dass er sich notwendig ergäbe. Dann wäre unser Wunsch kaum mehr als eine wohltätige Illusion. Und schnell abgetan.

\section{Leibniz als Zuflucht, als Burg?}

Doch haben alle, die nach einem anderen Konzept suchen, ihren Patron und Schutzheiligen in Leibniz. Er hat - auch mit seinem Gedankenexperiment den Weg veranschaulicht. Seine Einsicht könnte man so zusammenfassen: 
„Wer mit Methoden der Naturwissenschaft forscht, wird immer nur naturwissenschaftliche Vorgänge finden“. Er schreibt dazu im selben Abschnitt der Monadologie, das Denken sei „durch mechanische Gründe [... nicht erklärbar".

\section{Exkurs: Du Bois-Reymond und Hilbert}

Zu den Worten „durch mechanische Gründe [... n nicht erklärbar“ mache ich einen kleinen Exkurs und verweise auf Emil Du Bois-Reymond. Der bedeutende Berliner Physiologe hat im Jahr 1872 mit einem Vortrag höchstes Aufsehen erregt. Er erklärte das menschliche Bewusstsein als prinzipiell nicht erklärbar - nicht erklärbar aus den physiologischen Vorgängen im Gehirn. Dafür berief er sich auch auf das Mühlengleichnis von Leibniz. Und er endete in dem Ausruf: „Ignoramus, ignorabimus!“ (Wir wissen es nicht, wir werden es nicht wissen.) Eine Bankrotterklärung, sagten viele Kollegen, eine tiefe Erkenntnis, sagten andere.

Seine Tochter Estelle, die um 1912 seine Werke neu herausgegeben hat, lebte übrigens noch nach dem Zweiten Weltkrieg am Anfang des Hainholzweges, zusammen mit ihrer Schwester Rose, einer begabten Zeichnerin, ich habe beide noch gekannt. Wichtiger aber ist eine andere Verknüpfung mit Göttingen. Der bedeutendste unter den damaligen Mathematikern, David Hilbert, er starb hier 1943, war so empört über den vermeintlichen Verzicht des Kollegen Du Bois-Reymond auf Wissen, dass er sich, Sie kennen das, auf seinen Grabstein hier auf dem Friedhof an der Groner Straße trotzig die Worte setzen ließ: „Wir müssen wissen. Wir werden wissen“. Dieses Wort schleudert Hilbert damit auch Leibniz entgegen.

\section{Einerseits ganz Forscher... schroff daneben ein Metaphysiker}

Betrachten wir noch einmal das Mühlengleichnis. Leibniz kommt den anderen Wissenschaftlern seiner Zeit sehr entgegen, indem er die Natur als eine Maschine deutet. Seit Descartes ist die Maschine das bevorzugte Modell der Erklärung natürlicher Vorgänge. Das Modell Maschine diente der Absicht, behaupten zu können, alles sei mit Mechanik erklärbar und also determiniert. Leibniz greift das Modell gelassen auf. Er kann sogar seine Monaden als Maschinen beschreiben. Und besonders was das Gehirn angeht, da ist er ganz moderner Wissenschaftler und sagt: „Ja, das Gehirn ist eine Maschine wie eine Mühle“. 
Denn Leibniz konnte für sich in Anspruch nehmen, einerseits ein nüchterner und erfolgreicher Naturwissenschaftler zu sein, der dieser Wissenschaft mit der Infinitesimalrechnung das mathematische Rüstzeug gegeben hatte. Aber er war eben in beiden Welten zu Hause, in der Mechanik und in der Metaphysik. Und er konnte der Mechanik ihr Recht lassen, ohne die Metaphysik und den Geist aufzugeben. Doch die Metaphysik ist für ihn das Fundament.

Ja, - er meinte erkannt zu haben, dass es umgekehrt ist: Nicht das Geistige geht irgendwie aus der Materie hervor, sondern die Materie aus dem Geist! Die Mühle soll uns vor Augen führen, dass der menschliche Geist hier nicht entsteht. Die Erfahrung in der Mühle bestätigt damit, was Leibniz in einem Brief an einen Mathematiker schrieb, dass nämlich ,alles in den Naturerscheinungen gleichzeitig auf mechanische und auf metaphysische Weise geschieht, dass aber die Quelle der Mechanik in der Metaphysik liegt". Sehen können wir nur die Mechanik. Sie aber entsteht aus dem Geist.

\section{Zusammenfassung}

Warum wir Leibniz noch brauchen, darum sollte es gehen. Meine Antwort war: Weil Leibniz allen, die den Geist bewahren wollen, eine besonders kühne, radikale Strategie anbietet. Seine Auffassung von Geist und Materie ist kein Kompromiss, kein Rückzugsgefecht, keine Konzession, die bald überflüssig wird.

Er sagt nicht, was unsereiner vielleicht vertritt, der Geist entstehe „irgendwie" durch Sublimierung aus den biochemischen Vorgängen. Ja, selbst Du Bois-Reymond ist nicht so radikal wie er, denn der Berliner Physiologe meinte ja nur, wir würden es nie erkennen können, wie aus Molekularbewegungen Bewusstsein wird.

Leibniz setzt absolut auf den Geist. Nicht auf den Geist, der im menschlichen Gehirn entsteht, sondern auf den, den es schon immer gab, aus dem das Weltall besteht. Dieser Geist ist das Fundament! Und was unseren eigenen Geist angeht, so leitet der sich gar nicht aus der Physiologie des Gehirns ab.

Das ist die Umkehr des Gewohnten, eine radikale Position. Ich will sie nicht empfehlen. Vielleicht kann man sie heute nicht mehr vertreten, schon gar nicht mit allen Konsequenzen, die Leibniz in Kauf nimmt. Aber sie ist ein Leitbild, sie kann Mut machen. Denn so lange, wie der Geist durch einige moderne Forscher wegerklärt wird, kann uns Leibniz lehren, wie man die Geisthypothese wirklich begründet. Indem man den Geist des Universums zum Fundament macht - von allem. 


\section{Schluss}

Zum Schluss sei an einen anderen Außenseiter der Physik erinnert, an Johann Wolfgang Goethe und seine Farbenlehre. Über ihn als Naturwissenschaftler schrieb Carl Friedrich von Weizsäcker, und das will ich nun auf Leibniz angewandt wissen, diese schönen Worte: „Erst aus der Ferne erkennen wir, dass sein Licht nicht das des Leuchtturms ist, der den Hafen anzeigt, sondern das (Licht) eines Sterns, der uns auf jeder Reise begleiten wird“. 